\author{
Michael Noyong \\ Buelent Ceyhan \\ Christof M. Niemeyer \\ Ulrich Simon
}

\section{Preparation, structural, and optical features of two-dimensional cross-linked DNA/ gold-nanoparticle conjugates}

Received: 3 April 2006

Accepted: 2 May 2006

Published online: 25 May 2006

(C) Springer-Verlag 2006

M. Noyong · U. Simon $(\square)$

RWTH Aachen,

Institute of Inorganic Chemistry,

Landoltweg 1 ,

D-52074 Aachen, Germany

e-mail: ulrich.simon@ac.rwth-aachen.

de

B. Ceyhan - C. M. Niemeyer

Fachbereich Chemie,

Biologisch-Chemische

Mikrostrukturtechnik,

Universität Dortmund,

Otto-Hahn Str. 6

D-44227 Dortmund, Germany

\author{
Abstract The formation and \\ the optical features of two-dimen- \\ sional aggregates formed by \\ DNA-directed immobilization and \\ cross-linking of bifunctional \\ DNA-gold nanoparticles at flat \\ gold substrates are analyzed. The \\ samples are structurally character- \\ ized by atomic force microscopy \\ to evaluate the particle size, the \\ particle densities, and the degree \\ of aggregation. The optical \\ characteristics determined by UV/ \\ visible measurements are \\ correlated with the structural \\ features observed.
}

Keywords DNA · Nanoparticles · Self-assembly · Optical properties

\section{Introduction}

Noble metal nanoparticles exhibit size-dependent electronic and optical properties, which have gained much interests in material, life-, and nanosciences [1-4]. While the synthesis of such particles with different size and surface modifications is well established, there is still great demand in the arranging and self-assembling of the particles into ordered arrays to study the electronic and optical coupling between the particles as a function of the particle size and interparticle spacing [5-10]. The profound understanding of the different modes of interaction may finally lead to technical applications of such arrays, e.g., in nanoelectronic or sensing devices [3, 4, 11, 12].

In this context, DNA reveals excellent abilities for siteselective immobilization and ordering of metal nanoparticles in one to three dimensions [3, 12-16]. Our earlier studies focused on the DNA-directed immobilization (DDI) of gold particles leading to the formation of two-dimensional assemblies. These concepts showed the ability to bind, via the Watson-Crick base pairing, single-strand DNA-functionalized gold nanoparticles to complementary DNA single strands on surfaces. This ability was realized due to direct hybridization of complementary strands at the particles and surface, as well as by the use of linker molecules. The DDI concept was extended further to a multimolecular approach, which allows cross-linking between the immobilized particles $[14,17]$.

In this work, we describe the concept of DDI, together with the cross-linking of multifunctional DNA-functionalized gold nanoparticles to flat gold substrates. The structural features of the nanoparticle assemblies formed are analyzed by atomic force microscopy (AFM). These data will be related to the optical characteristics, based on the extinction properties recorded by bright field/reflection UV/visible (Vis) spectroscopy. These measurements are performed on reflecting gold surfaces, which allows us to analyze the optical features of the immobilized gold nanoparticles and particle aggregates in directed interaction with the underlying substrate.

Noble metal particles show a characteristic extinction in the visible range of the electromagnetic spectrum due to the excitation of plasmons. Plasmons are collective oscillations of surface electrons, which can be used for probing the vicinity of the particles' surfaces because of the sensitivity of the spectral position of the plasmon band to the particles' 
(dielectric) environment. Furthermore, the wavelength of the extinction maximum is dependent on the shape of the particles and on their spatial distance from each other. These extinction phenomena can quantitatively be described by the Mie theory, which relates the theoretical extinction spectra of diluted spherical particles to their size and relative dielectric properties of the surrounding medium [1]. As soon as particle aggregates are formed, the polarization needs to be described by the generalized Mie theory. When the center-to-center distance, $D$, of particles in an aggregate becomes as small as $D<<\lambda$, i.e., the wavelength of incident light, an in-phase coupling of the induced dipoles can be observed [15, 16, 18]. Compared to the single-particle resonance, the plasmon resonance of particle aggregates is shifted toward lower energies. The shift depends on the particles' distances and on the number of particles aggregated in the direction of the electrical field vector perpendicular to the incident light vector.

Thus, in case of a two-dimensional assembly of nanoparticles, the optical properties should give qualitative information about the degree of aggregation, indicating whether the particles are well separated from each other or whether they predominantly form aggregates. It is the aim of this work to qualitatively correlate the optical features of two-dimensional gold nanoparticle aggregates formed by DNA-directed immobilization and cross-linking to the structural features studied by AFM.

\section{Experimental}

Materials, particle synthesis, and particle assembly

Preparation of DNA-gold nanoparticle conjugates was carried out as previously described [13, 14, 19] using citrate-stabilized gold nanoparticles of $23.6 \pm 5.3 \mathrm{~nm}$ in diameter (ICN) and thiol modified DNA oligomers (Thermo Hybaid). In a typical synthesis, bifunctional DNA-Au nanoparticle conjugates were prepared by adding a mixture comprised of an equimolar ratio of the two oligonucleotides, e.g., 1 and 2 , to an aqueous nanoparticle solution $[14,20]$. For determination of surface coverages, 5'-fluorescein, 3'-( $\left.\mathrm{CH}_{2}\right)_{3}$-thiol-labeled oligonucleotides were used for preparing the DNA-nanoparticle conjugates [21]. The overall coverage was determined to about 260 oligonucleotides/particle. For bifunctional conjugates the amount of each oligonucleotide depends on the ratio of the respective mixture. The hybridization efficiency was at $20 \%$ coverage of one oligonucleotide at its maximum, which can be explained by the electrostatic repulsion of the single strands in higher concentrations. For detailed discussions of the results of the coverage and hybridization efficiency, see Niemeyer et al. [20].

The DNA/nanoparticle conjugates were purified by repeated centrifugation and redispersion in $0.3 \mathrm{M} \mathrm{NaCl}$, $20 \mathrm{mM}$ Tris- $\mathrm{HCl}, 5 \mathrm{mM}$ EDTA, and $0.05 \%$ Tween 20 , $\mathrm{pH}$ 7.5. Solid-phase hybridization experiments were carried out using flat gold substrates, prepared on mica as described earlier [22]. The substrates were functionalized with $5^{\prime}$ $\left(\mathrm{CH}_{2}\right)_{6}$-thiolated capture oligonucleotides (for base sequences, see Table 1) by incubation of a $10-\mu \mathrm{M}$ solution of the oligomer for $16 \mathrm{~h}$ at room temperature. The substrates were rinsed with deionized water and excess binding sites of the surface were blocked with a solution containing mercaptohexanol. In the trimolecular immobilization experiments with $\mathrm{D}_{2}-\mathrm{Au}$ conjugates, shown in Fig. 1, mixtures of linker oligomers 5 and 6 , each of which contained $0.3 \mu \mathrm{M}$ of 5 and varying concentrations of $6(0.3$ and $0.15 \mu \mathrm{M})$ in Tris-buffer containing $0.3 \mathrm{M} \mathrm{NaCl}$, were prepared. Of each mixture, $5 \mu \mathrm{L}$ was added to one aliquot of an equimolar mixture of $\mathrm{D}_{2}-\mathrm{Au}_{\mathrm{A}} / \mathrm{D}_{2}-\mathrm{Au}_{\mathrm{B}}(3 \mathrm{nM})$. The resulting solutions were briefly mixed and immediately dropped on top of a gold substrate, previously functionalized with capture oligomer 4. Following $6 \mathrm{~h}$ of incubation at room temperature, the substrates were rinsed with the Tris-buffer and deionized water and dried in a mild nitrogen stream.

\section{Preparation of substrates}

Atomically flat gold surfaces were prepared as follows: Glass slides were glued to a 20 -nm thin gold film, sputtered on a freshly cleaved mica sheet, and cleaved afterwards at the interface between the gold layer and the mica sheet. The pure gold surface was visualized by AFM, showing typical, flattened gold grains.

Table 1 Oligonucleotide sequences used in this study

\begin{tabular}{|c|c|}
\hline No. & Sequence \\
\hline 1 Particle-bound 12 mer for particle immobilization ${ }^{\mathrm{a}}$ & 5'-TTC GGA TCC CGG-SH-3' \\
\hline 2 Particle-bound 12 mer for cross-linking $\left(D_{2}-A u_{A}\right)^{a}$ & 5'-AAG ACC ATC CTG-SH-3' \\
\hline 3 Particle-bound 12 mer for cross-linking $\left(D_{2}-A u_{B}\right)^{a}$ & 5'-SH-GGT GAA GAG ATC-3' \\
\hline 4 Substrate-bound 12 mer capture oligomer ${ }^{\mathrm{a}, \mathrm{b}}$ & 5'-SH-TCG AAG CTT GAA-3' \\
\hline 5 Linker for trimolecular particle immobilization & 5'-CCG GGA TCC GAA TTC AAG CTT CGA-3' \\
\hline 6 Linker for particle cross-linking & 5'-CAG GAT GGT CTT GAT CTC TTC ACC-3' \\
\hline
\end{tabular}

${ }^{\mathrm{a}}$ The SH-functionalization has been realized by a $-\left(\mathrm{CH}_{2}\right)_{3}$-thiol at the $3^{\prime}$ end and a $-\left(\mathrm{CH}_{2}\right)_{6}$-thiol at the $5^{\prime}$ end

${ }^{\mathrm{b}}$ The sequence contained a dT12 spacer between the alkyl-thiol group and the coding sequence to improve hybridization efficiency 


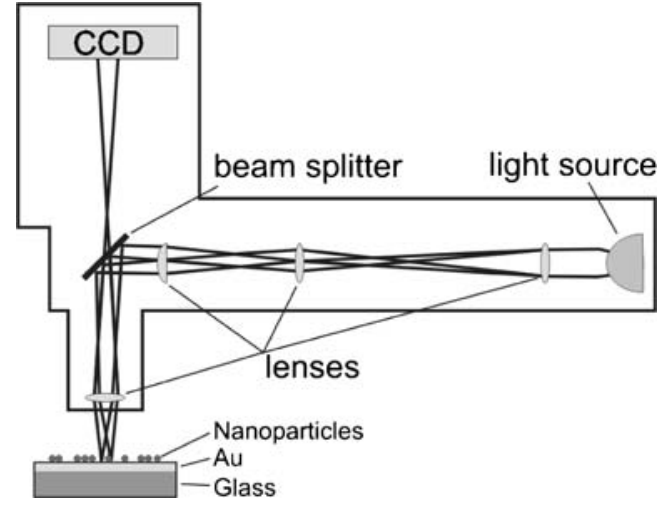

Fig. 1 Schematic optical path of the UV/Vis measurements at the reflecting gold surfaces with nanoparticles/particle aggregates

\section{Experimental setup}

AFM measurements were carried out using a Multimode/ Nanoscope IIIa from Digital Instruments in tapping mode in air at a relative humidity of about $40 \%$. We used Si cantilevers with a tip radius of approximately $5 \mathrm{~nm}$ (Nanosensors). AFM measurements may have suffered from tip artifacts, resulting in apparent enlargement of the objects studied. Such width anomalies are generally related to a nonlinear mixing of the tip shape into the image. Thus, the enlargement depends both on the tip radius and geometry and on the measured object itself [23-25]. Therefore, the size of the particles, as described in the following chapter, was obtained from height measurements.

The UV/Vis spectra were recorded in a range from 400 to $750 \mathrm{~nm}$ using a Zeiss Axioplan 2 imaging microscope (50-fold magnification) equipped with a UV/Vis diode array detector of J\&M, a halogen lamp as a light source, and a freshly prepared gold substrate as a reference. The measured spot on the sample was limited to $60 \times 40 \mu \mathrm{m}^{2}$. A scheme of the experimental setup is shown in Fig. 1.

\section{Results and discussion}

Our initial studies concerned the DDI of the $\mathrm{D}_{2}-\mathrm{Au}_{\mathrm{A}}$ and $\mathrm{D}_{2}-\mathrm{Au}_{\mathrm{B}}$ bifunctional nanoparticles, containing two $12 \mathrm{mer}$ coding sequences. To this end, gold substrates were functionalized by chemisorption of thiolated capture oligomer 4 . The DNA-modified gold substrate was used for immobilization of nanoparticles from a mixture, which contained equimolar amounts of $\mathrm{D}_{2}-\mathrm{Au}_{\mathrm{A}}$ and $\mathrm{D}_{2}-\mathrm{Au}_{\mathrm{B}}(3 \mathrm{nM}$, each), in addition to 16 molar equivalents $(\mathrm{eq})(0.3 \mu \mathrm{M})$ of linker 5 and $16 \mathrm{eq}(0.3 \mu \mathrm{M})$ of linker 6 . As schematically depicted in Fig. $2 b$, linker 5 was used to immobilize the $\mathrm{D}_{2}-\mathrm{Au}$ conjugates through hybridization with complementary capture oligomer 4 and particle-bound oligomer 1, while linker 6 was added to allow cross-linking in between the $\mathrm{D}_{2}-\mathrm{Au}_{\mathrm{A}}$ and $\mathrm{D}_{2}-\mathrm{Au}_{\mathrm{B}}$ particles, immobilized at adjacent sites of the substrate.
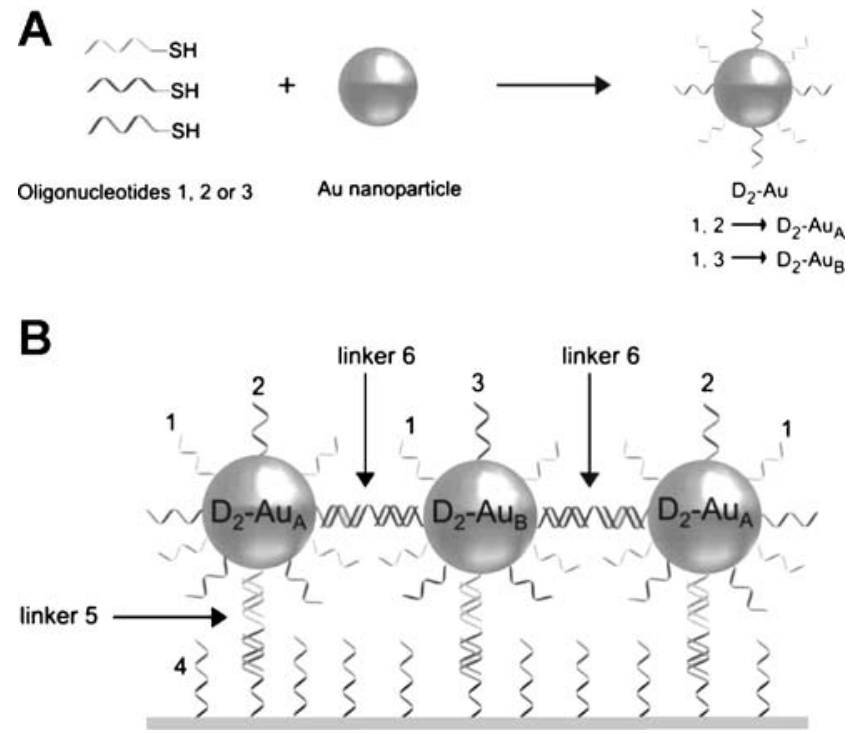

Fig. 2 Immobilization scheme. a Functionalization of the gold nanoparticles with oligomers. b Immobilization and cross linking of the particles onto the gold substrate

\section{AFM measurements}

AFM measurements were typically recorded from at least two separate representative areas of each sample with a size of a few square millimeters, preselected with an optical microscope to ensure a minimum number of impurities and defects. Figure 3 shows representative AFM images of four different samples. Figure 3 a shows a control which was just functionalized with the immobilization sequence 4 . The other three AFM images show the results of the immobilization of particles via the immobilization linker 5 and cross-linker 6 , in which the amount of the cross-linker was varied from 0 eq over 8 to 16 eq (Fig. 3c,d). The amount of immobilization oligomer 5 was held constant at 16 eq. To investigate the influence of the cross-linking oligomer, the sample without cross-linker 6 was used as a negative control. In the absence of cross-linker 6, the AFM image shows mainly well separated, statistically distributed single particles, and only very few aggregated particles (Fig. 3b). The samples with 8 and 16 eq 6 (Fig. 3c,d) show an increased number of aggregates. Height measurements of the immobilized nanoparticles revealed that these aggregates are, in fact, monolayers of particles and they are randomly shaped with lateral dimensions of up to $2 \mu \mathrm{m}$. The heights of the particles range from 15 to $24 \mathrm{~nm}$, which is found to be similar to the diameters determined by transmission electron microscopy, i.e., $23.6 \pm 5.3 \mathrm{~nm}$. Thus, the particles presumably lie very close to, or in direct contact with, the surface. Local order with hexagonal and cubic symmetry appeared locally in these aggregates, whereas no long-range order was observed. These results presumably are due to deviations in the particles' size and the surface roughness of 
Fig. 3 AFM images of DNAimmobilized gold nanoparticles with cross-linker DNA strands 6. a Gold surface functionalized with immobilization linker 4. b Immobilized gold nanoparticles with 16 eq of 5 , but without cross-linker 6 . Immobilized gold nanoparticles with 16 eq of 5 as well as 8 (c) and 16 eq (d) of cross-linker 6
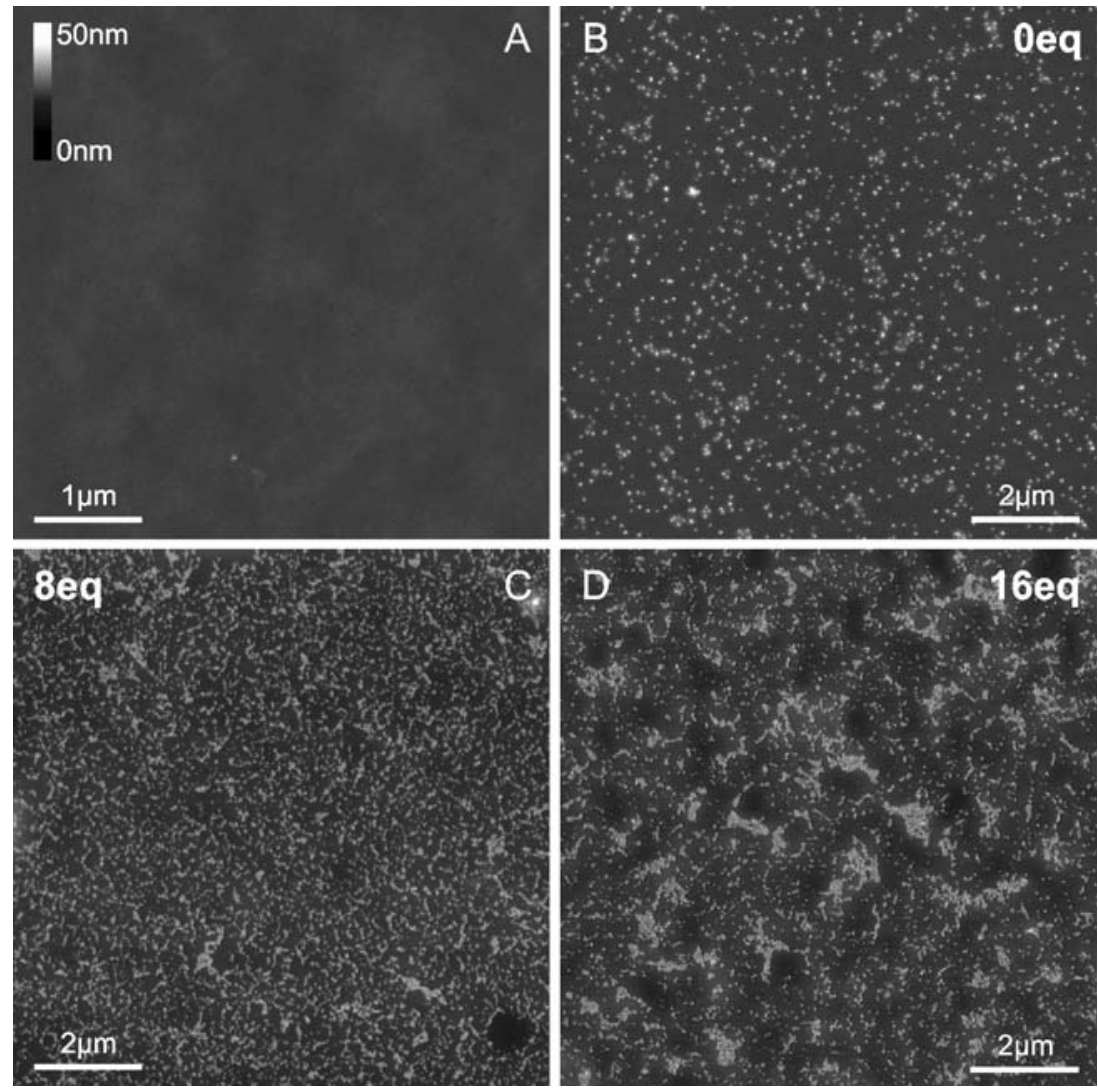

the underlying gold substrate, preventing the formation of highly ordered extended aggregates.

\section{Automated data analysis}

For the statistical evaluation of the immobilization process, 70 AFM images in total were recorded. To handle the huge amount of data, a computer program was developed, ${ }^{1}$ which automatically counted the particles in each AFM image and assigned them into aggregates in a two-pass process. The recognition of a particle was based on the gray scale in the AFM image, choosing the half of the average of the overall particle height as the criterion of selection (Fig. 4). To compensate the surface roughness and the particle size distribution, the program allows setting a percentage deviation, as well as minimal and maximal values for the expected diameters. This avoids the false detection of apparently big particles, which, indeed, are small aggregates of a few smaller particles. Particles, which are not recognized automatically, can be added manually based on the visual control of the selection process after the first pass of

\footnotetext{
${ }^{1}$ A demo version of the program can be applied from Dr. D. Sanders, Institute of Inorganic Chemistry, Rheinisch Westfälische Technische Hochschule (RWTH) Aachen, Landoltweg 1, 52074 Aachen. Contact e-mail: http://ulrich.simon@ac.rwth-aachen.de or http://daniel.sanders@ac.rwth-aachen.de
}

automated selection. Thus, the first pass gives the amount of particles per measured area, i.e., the average particle density.

In the second pass, the assignment of particles to aggregates is realized by the definition of a distance value, $d$, which is the expected value of the sum of two neighbored particles' radii and the length of the DNA linker. All those particles, which are located in distances equal to or smaller than $d$, are assigned to belong to an aggregate. Thus, the second pass leads to the aggregate size, which is not the lateral size but the number of particles per aggregate and the aggregate's size distribution.

For further data evaluation, all results were normalized to square micrometers and also converted to a percentage distribution. In general, in the presence of the cross-linker 6 , the immobilization rate is higher, which is already obvious in the AFM images in Fig. 3. As shown in Fig. 5, the particle density increases from 18 particles $/ \mu \mathrm{m}^{2}$ at 0 eq of 6 up to 260 particles $/ \mu \mathrm{m}^{2}$ at 8 eq of 6 , and decreases again to 153 particles $/ \mu \mathrm{m}^{2}$ in the presence of 16 eq of 6 . In contrast to the surface coverage, the maximum aggregate size (particles/aggregate) increased from 10 at 0 eq over 41 (at $8 \mathrm{eq}$ ) to 320 at $16 \mathrm{eq}$.

These results illustrate that in the absence of 6 , only a few particles form aggregates, probably due to nonspecific interaction. In all samples inspected by AFM, the particles formed smaller aggregates, whereas aggregates containing 
Fig. 4 Screenshot of the analysis program. a Imported image and $\mathbf{b}$ the processed AFM image. The different gray scales in $\mathbf{b}$ determine the particles, which are assigned as aggregates by the program

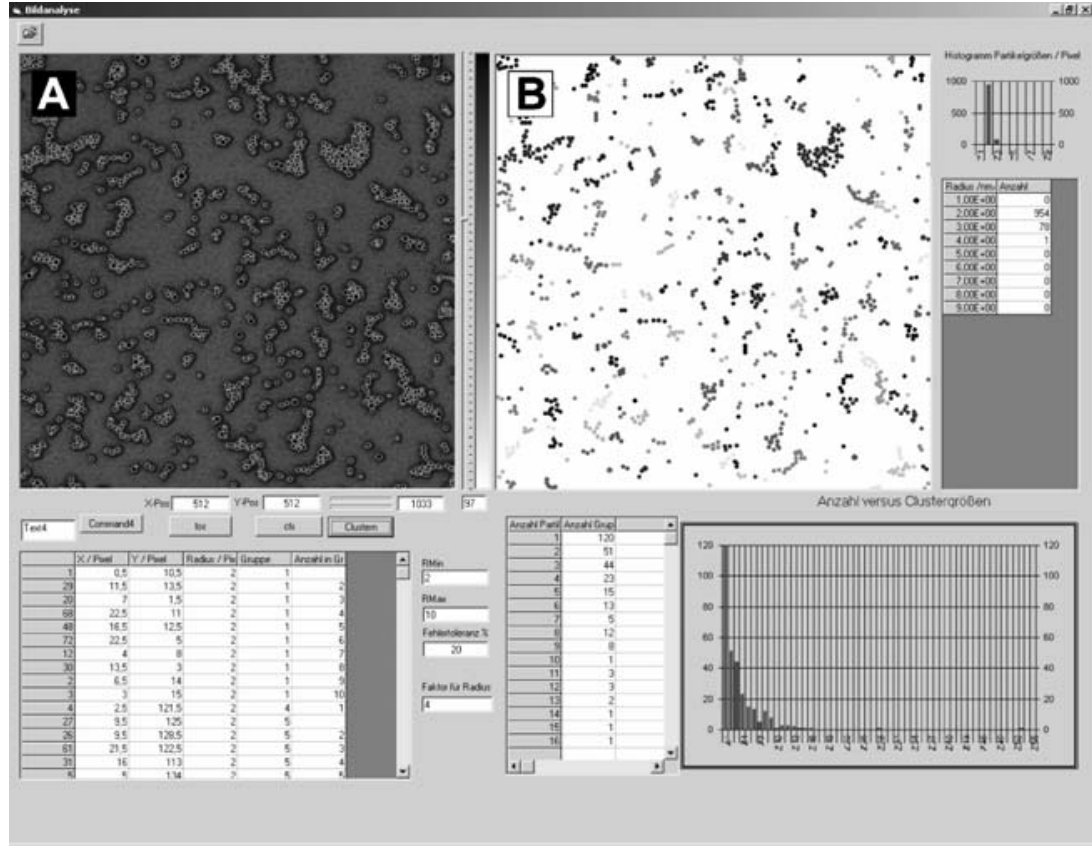

more than five particles were only observed in the presence of 6 , while samples without 6 showed the highest number of individual particles. The distribution of the aggregate's size in the presence of 8 and 16 eq of 6 was almost similar, whereas in the presence of 8 eq of 6 , slightly bigger amounts of aggregates with 2 and 10 particles/aggregate were found. The largest aggregates, containing more than 300 particles per aggregate, were found in samples containing 16 eq of 6.

Because there is no direct experimental proof as to whether or not the nanoparticles are indeed connected via the DNA cross-linker, we determined the distance between individual particles within the aggregates and compared it to the theoretical length of the cross-linker 6 , i.e., $9.6 \mathrm{~nm}$. Because the distances between the particles cannot be measured by AFM directly, the particle distance was deduced from the distance of the height maxima of the imaged particles. To ensure that the particle size distribution did not affect these results, the distance between the particles was corrected by the respective particle diameters $\delta_{1}$ and $\delta_{2}$. Therefore, for each evaluated pair of particles, the radius, $H$, of each individual particle was determined from the maximum height of a particle in a cross section (see Fig. 6).

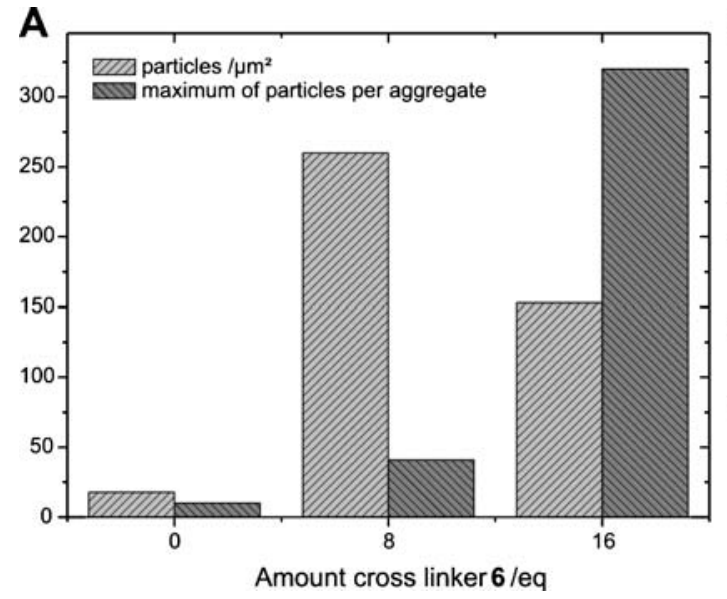

Fig. 5 Results of the automated analysis of particle density and particles per aggregate. a Particle density and maximum size of aggregates plotted vs ratio of cross linker 6 . The immobilizing linker was held constant at 16 eq. b Percentage amount of aggregate sizes

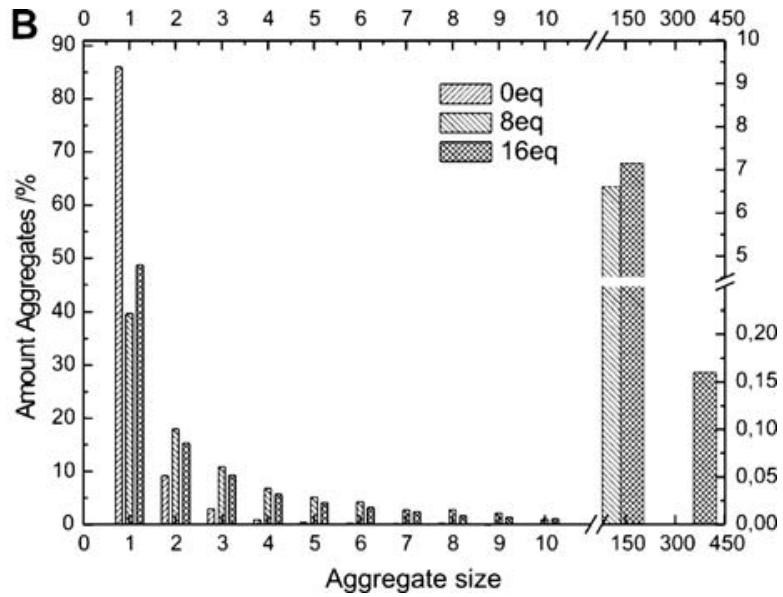

per square micrometer. For aggregate sizes up to ten particles per aggregate, the amount is displayed on the left y-scale, whereas the summarized range of aggregate sizes from 11 to 100 and 101 to 320 refer to y-scale on the right-hand side 
With $H$, the effective distance between the particles, $d_{\text {eff }}$, can be calculated by measuring $X$, i.e., the top-to-top distance $d_{\text {center1-center2 }}$, according to

$$
d_{e f f}=X-H=d_{\text {center } 1-\text { center } 2}-\frac{\left(\delta_{1}+\delta_{2}\right)}{2}
$$

The distance distribution for the samples with 8 and 16 eq of 6 is plotted in Fig. 7. By taking the calculated value $d_{\text {crosslinker }}=9.6 \mathrm{~nm}$, which is the sum of the DNA length and that of thioalkyl-linkers at the $5^{\prime}$ and $3^{\prime}$ ends of nanoparticle-bound oligomers 2 and 3, and taking into account a fault tolerance value $f_{\max }=1.3 \mathrm{~nm}$, resulting from the maximum surface roughness $r_{\text {substrate }}$ and from the limits of resolution $f_{\text {res }}$, it becomes evident that $47 \%$ (8 eq) and $41 \%$ (16 eq) of all measured pairs of particles have an effective distance $d_{\text {eff }}$, which is close to the expected value of $9.6 \pm 1.3 \mathrm{~nm}$. Furthermore, for both systems, $6.8 \%$ (8 eq) and $6.9 \%$ (16 eq) of the pairs of particles show smaller distances. This implies that the cross-linker has certain flexibility and/or that the cross-linker does not connect the particles along the center-to-center axis between two particles. Of the particles, $46.4 \%$ (8 eq) and 51.9\% (16 eq) show distances to their neighbors that are much longer than $d_{\text {crosslinker6, }}$ implying that these particles are not connected by 6 or that mismatches in the DNA hybridization have led to a longer effective molecule length.

\section{Optical properties}

The optical characterization of these samples required measurements in reflection because the sputtered gold layers were too thick for measurements in transmission mode. Already, the observation of the substrates with immobilized particles by the naked eye shows, in contrast to the bright golden reflection of an unfunctionalized surface, a slight red color. For the UV/Vis spectra recorded in reflection on the nontransparent gold films, the total reflection of the light source on a bare gold substrate without particles was taken as a reference. Thus, in spectra taken from the samples with immobilized particles, we observed the particles' extinction, resulting from absorbance as well as from scattering [1]. As is known from earlier studies, for individual particles smaller than $20 \mathrm{~nm}$, scattering can be neglected [1], while the extinction of aggregates results from a superposition of scattering and absorbance [5].

The UV/Vis spectra of the immobilized particles (Fig. 8) revealed pronounced differences to the spectra of single particles in solution. The extinction at $520 \mathrm{~nm}$, well known for single, well-separated particles in solution, is missing in the spectra shown here. However, an extinction at about $500 \mathrm{~nm}$ was observed instead. Furthermore, an additional peak at about $700 \mathrm{~nm}$ appeared for those samples that showed particle aggregates.

First, the loss of the peak at $520 \mathrm{~nm}$ was unexpected, but may be explained by a blue-shift of the plasmon resonance of the metal nanoparticles in close vicinity to the metallic substrate. This effect was described before as a result of a hybridization of the surface plasmon of the metallic substrate with the particles' plasmon [26, 27]. Therefore, we tentatively assign the absorbance appearing at about $500 \mathrm{~nm}$ to the plasmon resonance of isolated gold particles affected by the metallic substrate.

Second, the two samples with 8 and 16 eq of 6 showed an additional extinction maximum at 690 and $700 \mathrm{~nm}$, respectively. The appearance of such extinction at longer wavelengths has repeatedly been observed in measurements of gold nanoparticles in layers with high particle density and of particle aggregates, respectively [1, 18, 28-30]. According to this, we attribute the red-shift to the coupling of the
Fig. 6 a Magnified part of the AFM image in Fig. 3d. b Measured height of the AFM tip along the path shown in a as a function of the displacement $x$. The horizontal doted lines illustrate the height value at the particle maximum, while the vertical dotted lines illustrate the displacement at their maxima. c Sketch of a particle pair, which is spaced by a linker, and an AFM tip. The bold line shows the cross section, from which $X$ (the center-to-center distance of the particles) and $\delta$ (diameters of the particles) can be determined. $\mathbf{d}-\mathbf{f}$ In addition to $\mathbf{a}$, further examples of AFM images with chosen pathways
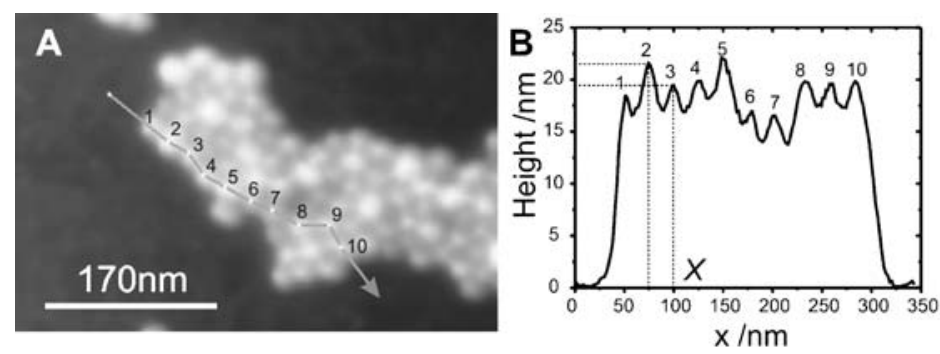

C

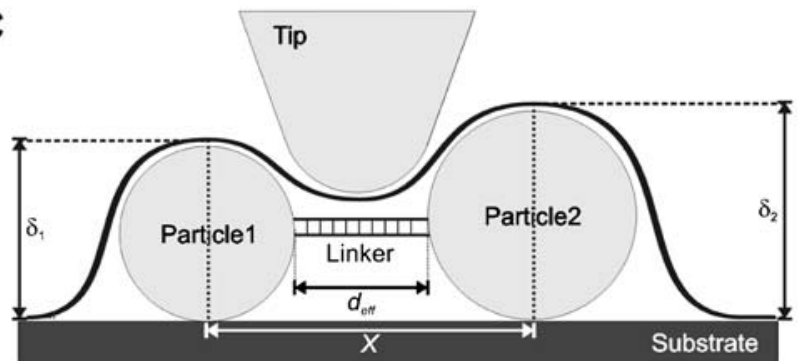

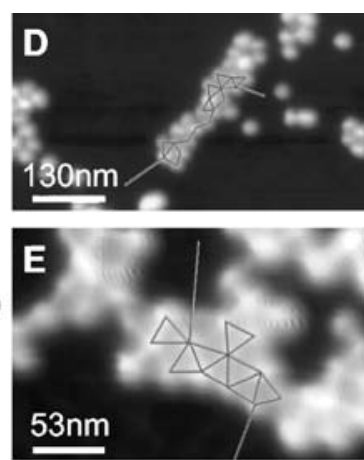

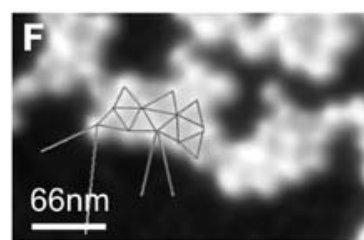


Fig. 7 Analysis of the distance between particles (see cross section in Fig. 6) for the two samples with cross linker 6 (a with 8 eq and b with $16 \mathrm{eq}$ ). The values of 237 and 291, respectively, show the amount of analyzed distance. On the right-hand side of each histogram, a summarized amount of $d_{\text {eff }}$ for smaller, even, or bigger values than the expected value of $d_{\text {crosslinker6 }}$ is given

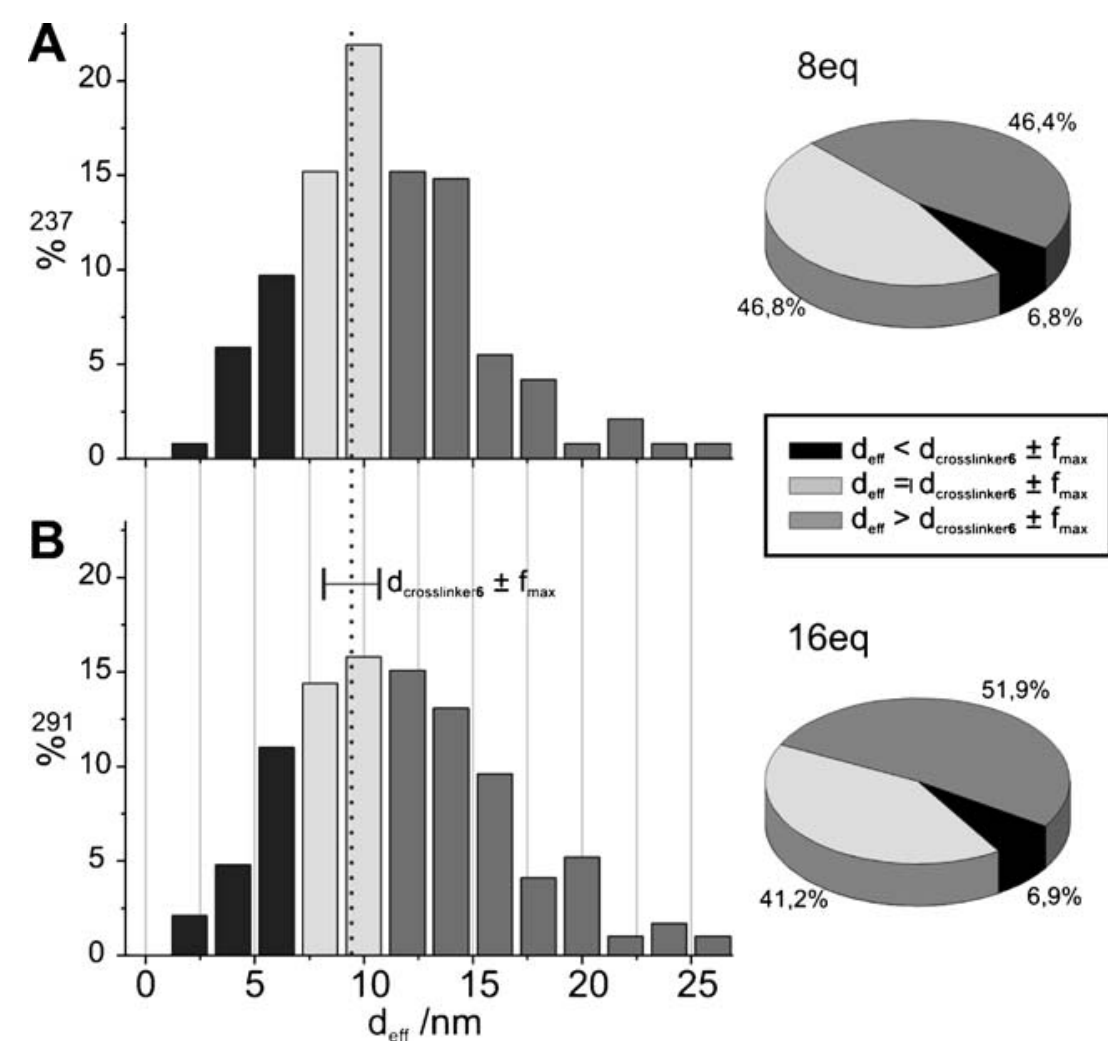

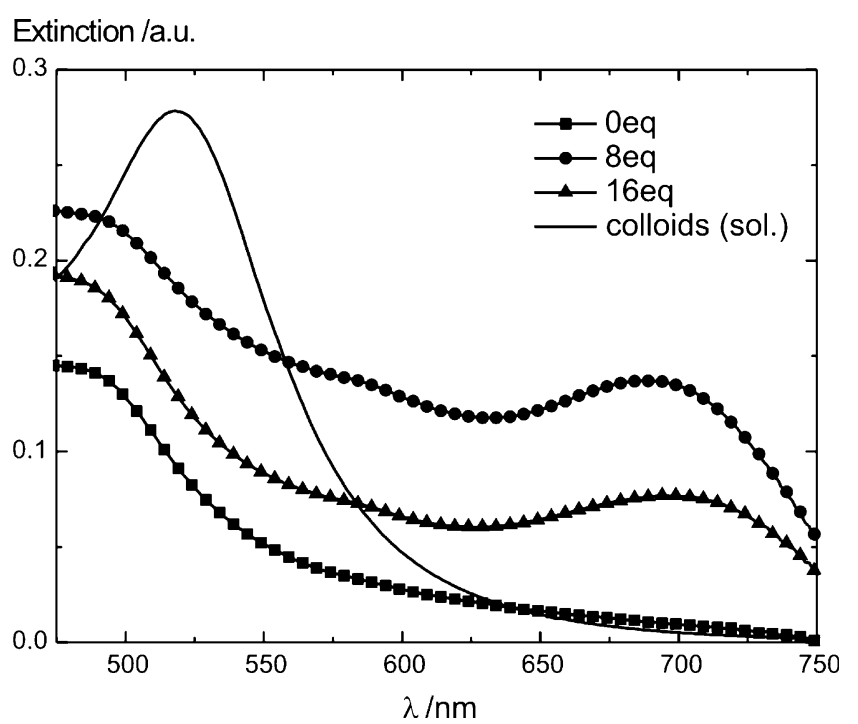

Fig. $8 \mathrm{UV} /$ Vis measurements of particle layers with different amounts of cross linker. Additionally, the spectrum of particles in aqueous solution is shown

plasmon resonance of particles close to each other, forming longitudinal in-phase modes. The degree of the shift depends mainly on the size of the particles, the particle distances, and the shape of the aggregates.

In the following, some results from literature shall shortly be reviewed to compare them with our experimental find- ing. Schmitt et al. observed the appearance of two plasmon peaks at 518 and $610 \mathrm{~nm}$ for 14-nm gold particles immobilized on a glass substrate via poly(ethylene-imine) [18], while Grabar et al. measured two, not-well-separated peaks at 536 and $648 \mathrm{~nm}$ for 13-nm gold particles immobilized on an amino-terminated silanized quartz slide (via 3-aminopropyltrimethoxysilane) [29]. Kreibig and Vollmer reported about two plasmon peaks at 520 and $700 \mathrm{~nm}$, measured from 15-nm gold particles embedded in a gelantine matrix with an average center-to-center distance of $18 \mathrm{~nm}$ [1]. Furthermore, Lazarides and Schatz calculated optical spectra for linear aggregates of 13-nm gold spheres of different lengths (up to ten spheres) [5]. They showed that, with increasing chain lengths, the plasmon peak is shifted toward lower energies. For chains consisting of ten particles, the maximum was observed at approximately $630 \mathrm{~nm}$. Experimental and theoretical results of ordered nanoparticle arrays (gold cylinders with diameters of $200 \mathrm{~nm}$ ) immobilized at glass slides showed the pronounced extinction in the range of 700 to $800 \mathrm{~nm}$ [31].

However, the low-energy shift for particles with comparable diameters and interparticle spacing, as observed here, is more pronounced than it was reported in the references cited above. Here, again, one needs to take into account that our nanoparticles were not immobilized on a dielectric surface (i.e., glass) but on a metallic substrate, where the dipolar and, possibly, additional multipolar coupling to the underlying gold surface might enhance this effect. This assumption is supported by the results of Félidj 
et al., who reported an enhanced substrate-induced coupling of gold nanoparticles in defined arrays on a gold surface through surface-plasmon traveling waves [28]. While the particle spacing was decreased, a shift of extinction maximum toward lower energies was observed. Nevertheless, this is just a qualitative measure on how a metallic substrate might affect the plasmon peak of the nanoparticles.

However, the development of a more refined model is not possible based on the results shown here. As far as we know, a full theoretical treatment of the longitudinal and transversal absorption of large particle aggregates including the coupling to metallic substrate has not been elaborated yet, due to the relatively complex geometry of the problem. Nevertheless, the separation of the single particle peak and the transversal extinction band, which assign to particles in aggregates, is expected to be more pronounced, the more extended the aggregates are.

As an additional feature in the optical spectra, we observed that the total optical extinction increased with increasing particle density. According to the Lambert-Beer law, the extinction correlates to the number of particles per volume or square unit. To verify the assumption that both plasmon peaks can be attributed to the optical extinction of individual particles and to particles within aggregates, respectively, we related the number of isolated particles to the absorbance at $500 \mathrm{~nm}$, and the number of particles in aggregates (two or more particles with $d_{\text {eff }} \leq d_{\text {crosslinker6) }}$ ) to the respective low-energy maximum. For the samples without cross-linker 6, we choose the extinction at $690 \mathrm{~nm}$ for this evaluation. This is justified by the fact that the decay of the plasmon peak in the spectra of particles in solution between 650 and $730 \mathrm{~nm}$ is more pronounced than in the spectra of the particles on the substrate. The extinction of particles in solution converges in this range to zero, while the samples without cross-linker showed an almost linear decrease with a remaining weak extinction, which is assigned to the plasmon resonance in aggregates.

A more detailed comparison was done by partitioning the particle density in (a) single particles/small aggregates and (b) aggregates (see Fig. 9a). Besides, both graphs start at zero to regard the fact that a particle density of zero cannot show any extinction. The results are displayed in Fig. 9b.

It is evident that the particle densities scaled to one square micrometer and the extinctions at the high- and lowenergy peaks show a linear correlation. The best linear fit for the high-energy peaks was achieved for partition A(1) of single particles. This means that the absorption of mainly single particles is attributed to the extinction at $500 \mathrm{~nm}$. The best linear fit for the extinction at about $700 \mathrm{~nm}$ can be achieved by choosing the particle density B (2-5) of small particle aggregates with $2-5$ particles per aggregate. However, correlation is also found for larger aggregates (e.g., with 2-320 particles per aggregate), but the accordance is less pronounced. The linearity is expected from the Lambert-Beer law, whereas the slope would give the molar extinction coefficient. However, the quantitative evaluation is not appropriate due to the evident influence of the underlying substrate, as discussed before. Although this correlation does not serve as physical proof, it supports our assignment of the peaks in the optical spectra, and it is in accordance with the experimental findings reported by others $[1,5,18,26-30]$.

\section{Conclusions}

We have analyzed the formation and the optical properties of two-dimensional aggregates formed by DDI and crosslinking of bifunctional DNA-gold nanoparticles at flat gold substrates. The particle density in the two-dimensional aggregates is controlled by the amount of the cross-linking oligonucleotides. This density as the degree of aggregation is reflected in the extinction of the optical spectra by the appearance of a low-energy plasmon resonance. This extinction peak could tentatively be attributed to longitudinal in-phase modes of the plasmon excitation. Furthermore, an extinction maximum at $500 \mathrm{~nm}$ could be observed, which has been assigned to a blue-shift of the plasmon peak of individual nanoparticles caused by coupling to the metallic substrate. So the results from the statistical examination
Fig. 9 a Scheme for the partitioning of the overall particle density into $(A)$ single particles/ small aggregates and $(B)$ aggregates. The numbers in brackets denote the number of particles per aggregate. b Plot of the extinction at $500 \mathrm{~nm}$ and 650 $730 \mathrm{~nm}$ vs the particle densities of $A(1)$ single particles and $B(2-$ $5)$ aggregates (the lines shown here just serve as a guide for the eye)
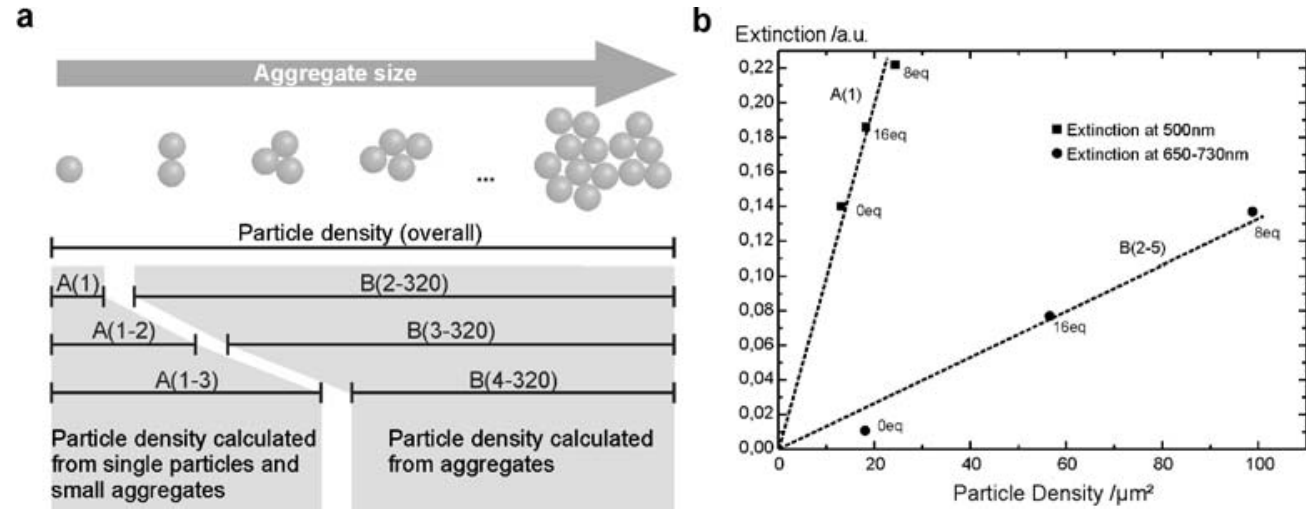
could be correlated with features in the optical spectra. Although these results of the optical investigation of gold nanoparticles on gold surfaces are very promising, further investigation of the interaction between the particles and the metallic substrate, as well as a possible quantitative correlation, needs to be developed in our future work.
Acknowledgements We thank Prof. Dr. U. Kreibig, Prof. Dr. G. von Plessen and C. Dahmen from the Department of Physics at the RWTH Aachen for their fruitful discussions about the optical data. We also thank Dr. D. Sanders from the Institute of Inorganic Chemistry at the RWTH Aachen for the analysis program. This work was supported by Deutsche Forschungsgemeinschaft (DFG) in Schwerpunktprogramm 1072 through grants Ni399/4-1-3 (CMN) and Si609/3-1 and 4-1,2 (US).

\section{References}

1. Kreibig U, Vollmer M (1995) Optical properties of metal clusters. Springer, Berlin Heidelberg New York (and references therein)

2. Schmid G (2003) Nanoparticles. Wiley-VCH, Weinheim

3. Niemeyer CM (2001) Angew Chem Int Ed Engl 40:4128

4. Katz E, Willner I (2004) Angew Chem 116:6166

5. Lazarides AA, Schatz GC (2000) J Phys Chem B 104:460

6. Wuelfing WP, Murray RW (2002) J Phys Chem B 106:3139

7. Torma V, Vidoni O, Simon U, Schmid G (2003) Eur J Inorg Chem 1121

8. Wessels JM, Nothofer HG, Ford WE, Wrochem F, Scholz F, Vossmeyer T, Schroedter A, Weller H, Yasuda A (2004) J Am Chem Soc 126:3349

9. Koplin E, Niemeyer CM, Simon U (2006) J Mat Chem 16:1338

10. Juillerat F, Solak HH, Bowen P, Hofmann H (2005) Nanotechnology 16:1311
11. Schmid G, Simon U (2005) Chem Commun 6:697

12. Niemeyer CM, Simon U (2005) Eur J Inorg Chem 18:3641

13. Niemeyer CM, Ceyhan B, Gao S, Chi L, Peschel S, Simon U (2001) Colloid Polym Sci 279:68

14. Niemeyer CM, Ceyhan B, Noyong M, Simon U (2003) Biochem Biophys Res Comm 311:995

15. Storhoff JJ, Lazarides AA, Mucic RC, Mirkin CA, Letsinger RL, Schatz GC (2000) J Am Chem Soc 122:4640

16. Storhoff JJ, Mirkin CA (1999) Chem Rev 99:1849

17. Zou B, Ceyhan B, Simon U, Niemeyer CM (2005) Adv Mater 17:1643

18. Schmitt J, Mächtle P, Eck D, Möhwald H, Helm CA (1999) Langmuir 15:3256

19. Storhoff JJ, Elghanian R, Mucic RC, Mirkin CA, Letsinger RL (1998) J Am Chem Soc 120:1959

20. Niemeyer CM, Ceyhan B, Hazarika P (2003) Angew Chem Int Ed Engl 42:5766

21. Demers LM, Mirkin CA, Mucic RC, Reynolds RA, Letsinger RL, Elghanian R, Viswanadham G (2000) Anal Chem $72: 5535$
22. Niemeyer CM, Ceyhan B, Gao S, Chi L, Peschel S, Simon U (2001) Colloid Polym Sci 279:68

23. Bustamante C, Vesenka J, Tang CL, Rees W, Guthold M, Keller R (1992) Biochemistry 31:22

24. Ikai A (1996) Surf Sci Rep 26:261

25. Gao S, Chi L, Lehnert S, Ancykowski B, Niemeyer CM, Adler M, Fuchs H (2001) Chem Phys Chem 2:384

26. Nordlander P, Prodan E (2004) Nano Lett 4:2209

27. Ruppin R (1992) Phys Rev B 45:11209

28. Félidj N, Aubard J, Levi G, Krenn JR, Schider G, Leitner A, Aussenegg FR (2002) Phys Rev B 66:245401.1

29. Grabar KC, Freeman RG, Hommer MB, Natan MJ (1995) Anal Chem 67:735

30. Bockstaller MR, Thomas EL (2004) Phys Rev Lett 93:166106/1

31. Hayes CL, McFarland AD, Zhao L, van Duyne RP, Schatz GC, Gunnarson L, Prikulis J, Kasemo B, Käll M (2003) J Phys Chem B 107:7337 